\section{International Scientific Journal Theoretical \& Applied Science}

p-ISSN: $2308-4944$ (print)

e-ISSN: 2409-0085 (online)

Year: 2015

Issue: 02

Volume: 22

Published: 28.02.2015

http://www.T-Science.org
Alexandr Nikolayevich Shevtsov candidate of technical sciences, member of PILA (USA),

Department of «Mathematics»,

Deputy Director on Science of faculty of information technologies, automation and telecommunications, Taraz state University named after M.Kh. Dulati,

Kazakhstan

$\underline{\text { Shev AlexXXXX@mail.ru }}$

SECTION 2. Applied mathematics. Mathematical modeling.

\title{
THE COMPARISON ALGORITHM DOCUMENTS
}

Abstract: While handling the documents of a significant amount of is often a problem comparing them to match before and after editing. For tasks, this work describes a simple and effective code for Delphi Architect allows such an analysis of the documents in Word format and calculate the percentage of matches.

Key words: Delphi, Word, match, algorithm.

Language: Russian

Citation: Shevtsov AN (2015) THE COMPARISON ALGORITHM DOCUMENTS. ISJ Theoretical \& Applied Science 02 (22): 16-20. doi: http://dx.doi.org/10.15863/TAS.2015.02.22.4

\section{ОБ ОДНОМ АЛГОРИТМЕ СРАВНЕНИЯ ДОКУМЕНТОВ}

Аннотация: При обработке документов значительного объема зачастую возникает проблема их сравнения на совпадения до и после редактирования. Для поставленной задачи, данная работа описывает простой и эффективный программный код на Delphi Architect позволяющиий производить подобный анализ документов в формате Word и рассчитывать процент совпадений.

Ключевые слова: Delphi, Word, совпадение, алгоритм.

Сравнение документов достаточно актуальная проблема описываемая в различных источниках [1-8]. Сравнивать документы вручную - занятие неблагодарное из-за слишком больших затрат времени и возможности ошибок, ведь не заметить какую-то важную деталь при просмотре проще простого. Гораздо разумнее задачу сравнения файлов перепоручить компьютеру [1].

1. Сравнение непосредственно в Word.

В целом, в плане сравнения Wordдокументов все обстоит достаточно неудобно, хотя и без использования вспомогательных инструментов, в Word данная возможность надежно скрыта от чужих глаз, и, вероятно, не так много пользователей о ее существовании вообще догадываются [1]. Результат сравнения окажется представленным в новом документе, где слева будет отображен отрецензированный документ с учетом изменений, а справа (друг над другом) - исходный и измененный документы.

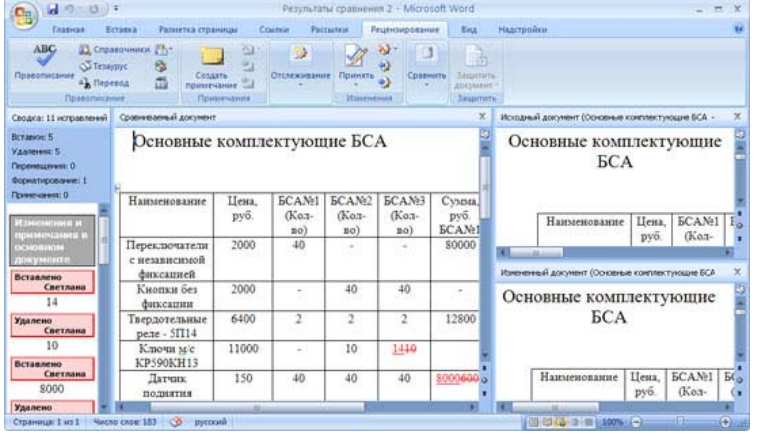

Рисунок 1 - Сравнение документов непосредственно в Word.

Tеоретически, в Excel тоже возможно сравнение документов встроенными средствами, правда, только при работе в режиме фиксирования изменений. Однако это неудобно, поскольку каждую из измененных ячеек придется просматривать, наводя на нее мышь, так как изменения, внесенные в документ, отображаются во всплывающих окошках (примерно таких, как обычные примечания) [1].

2. Сравнение в Compare Suite 7.0 компании AKS-Labs. 
Достаточно удобный инструмент для быстрого сравнения текстовых файлов, документов MS Office, RTF-документов, файлов PDF, webстраниц (HTM), презентаций PowerPoint, бинарных и некоторых других типов файлов, а также файлов в ZIP- и RAR-архивах и на FTPсерверах. В случае сравнения листингов программ на ряде языков программирования (Object Pascal, HTML, C/C++, JavaScript, PHР и др.) предусмотрена подсветка синтаксиса. Имеется функционал для синхронизации текстовых файлов и сравнения папок вместе с подпапками [2].

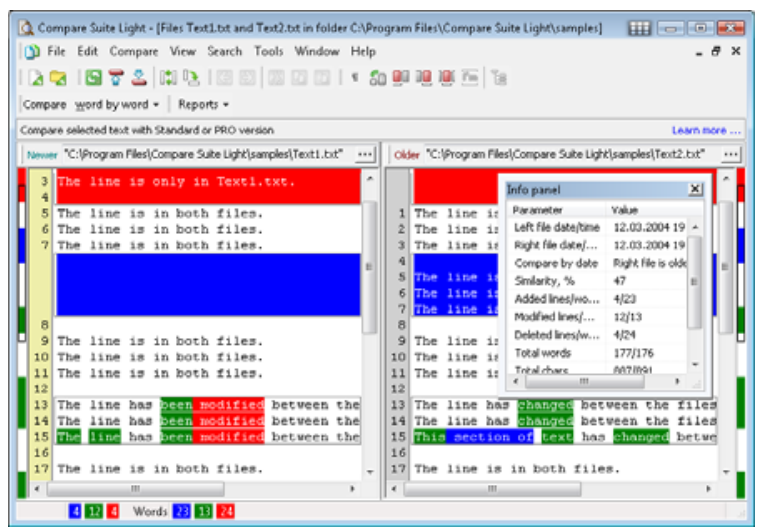

Рисунок 2 - Сравнение в Compare Suite.

Кроме этого комплексы сравнения также представленны следующими разработками:
3. Compare It! 4.1.2174 [3];
4. Beyond Compare 3.1.9 [4];
5. WinMerge 2.12.4 [5];
6. Active File Compare 2.0 [6];
7. Compare Spreadsheets for Excel 1.1.5 [7];
8. Excel Compare 2.3 [8];
9. xc Excel Compare Tools 1.0 [9];
10. Spreadsheet Compare 1.15 [10];

Приведенные программы обладая широким функционалом, неудобны в использовании, когда необходимо с минимумом совершаемых действий получить результат. А при смещении всего на 1 символ выдают абсолютно неверные результаты, так как основаны на побуквенном сравнении.

Как результат - они абсолютно неприменимы, например при анализе двух экзаменационных тестов на повторяющиеся вопросы. Необходима разработка более совершенных алгоритмов сравнения документов.

В основе нашего алгоритма будем использовать разбиение одного из документов на блоки и поблочное сравнение.

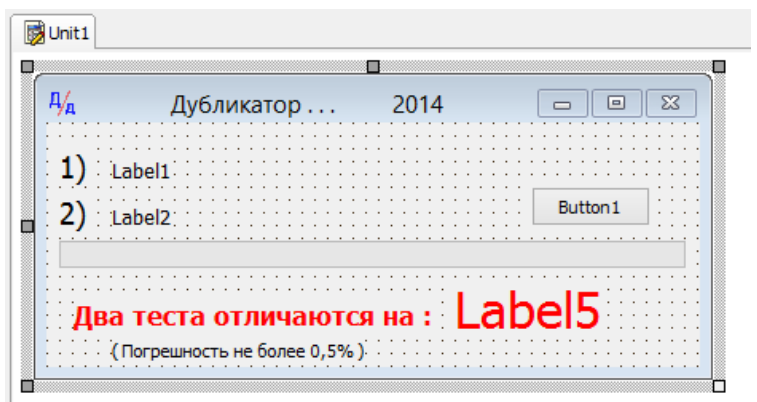

Рисунок 3 - Форма программы.

Разработаем алгоритмы и программный код на Delphi Architect. Загрузка документов будет осуществляться методом Drag\&Drop, при последующей автоматической перезагрузке, и возможности не закрывая программу сразу же после анализа двух тестов перейти к анализу двух других. Действия пользователя сводятся к перетаскиванию двух файлов на форму программы, что значительно ускоряет и оптимизирует работу.

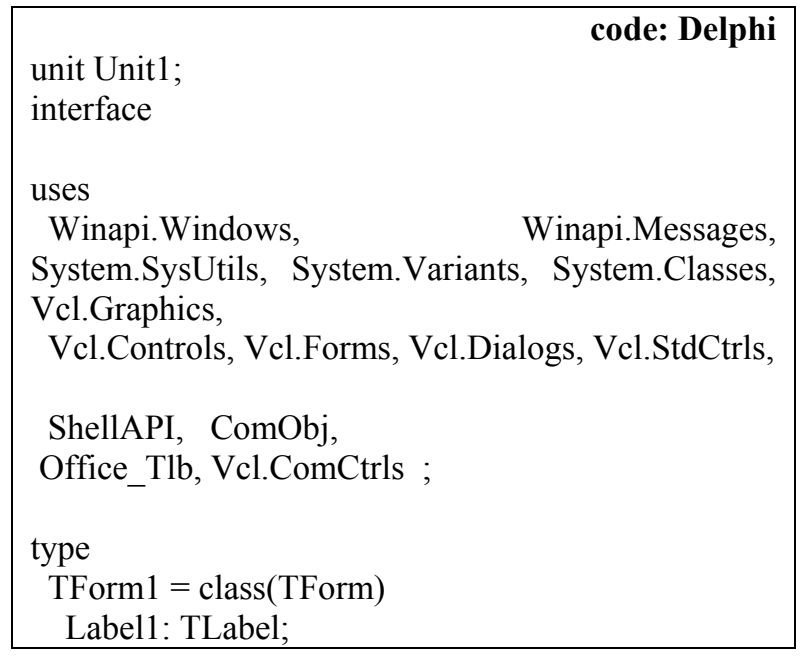

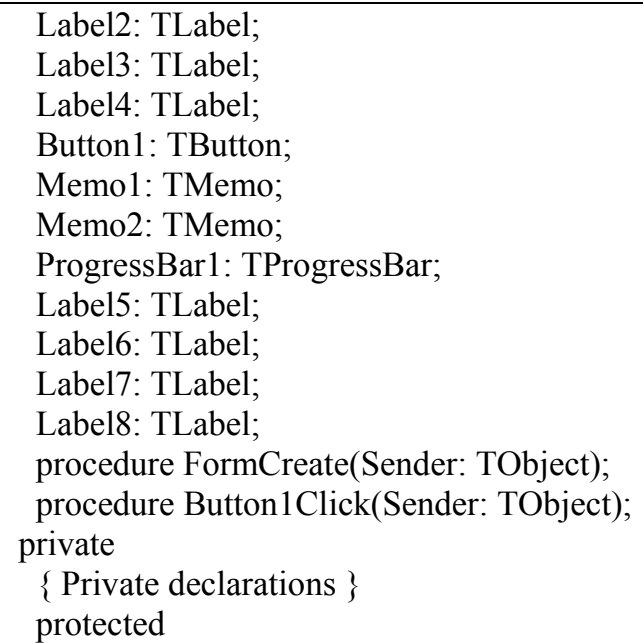




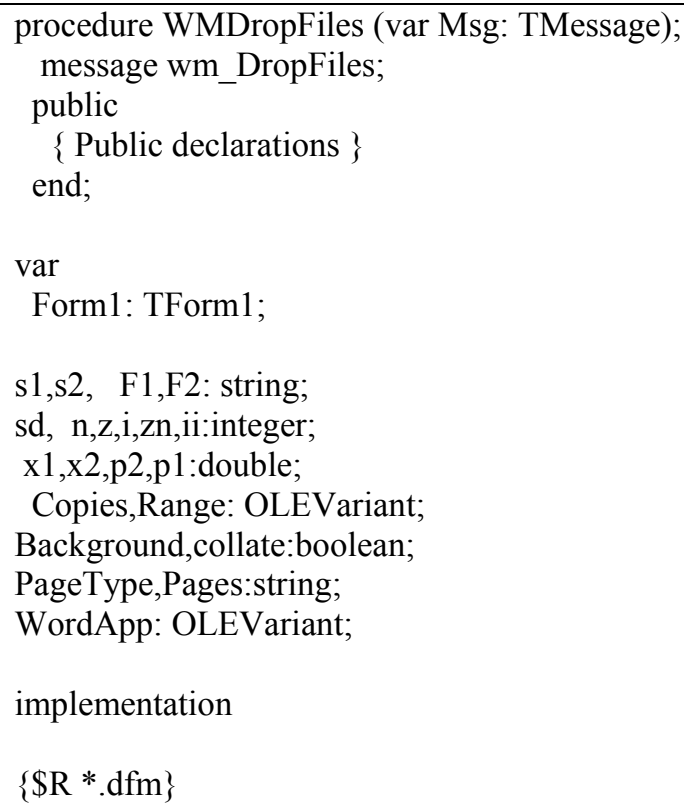

WordApp.ActiveDocument.Close(true,EmptyParam, EmptyParam);

WordApp.Documents.Open(f2);

memo2.Text:=WordApp.ActiveDocument.Range.Te $\mathrm{xt}$;

WordApp.ActiveDocument.Close(true,EmptyParam, EmptyParam);

$\mathrm{sd}:=50 ; \quad$ // коэффициент сравнения

$\mathrm{s} 1:=$ memo1.Text;

$\mathrm{s} 2:=$ memo2.Text;

$\mathrm{zn}:=\operatorname{trunc}($ length$(\mathrm{s} 1) / \mathrm{sd})$;

progressbar1.Position: $=0$;

progressbar1.Max:=zn;

$\mathrm{z}:=0$

for $\mathrm{I}:=1$ to $\mathrm{zn}$ do

begin

if $\operatorname{pos}\left(\operatorname{copy}\left(\mathrm{s} 1, \mathrm{i}^{*} \mathrm{sd}, \mathrm{sd}\right), \mathrm{s} 2\right)>0$ then inc $(\mathrm{z})$; progressbar1.Position: $=\mathrm{i}$;

application.ProcessMessages;

end;

$\mathrm{x} 1:=\operatorname{int}((\mathrm{zn}-\mathrm{z}) / \mathrm{zn} * 10000) / 100$;

ii: $=$ zn;

$\mathrm{s} 1:=$ memo2.Text;

s2:=memo1.Text;

$\mathrm{zn}:=$ trunc(length $(\mathrm{s} 1) / \mathrm{sd})$;

progressbar1.Position: $=0$;

progressbar1.Max:=ii+zn;

label7.Caption:=floattostr(ii)+' '+floattostr(zn);

p1:=300/ii;

p2:=300/zn;

if $\mathrm{p} 1>\mathrm{p} 2$ then $\mathrm{p} 2:=\mathrm{p} 1$;

label8.Caption: $=$ ' $($ Погрешность не более

'+floattostr(int(p2*100)/100)+' \% )';

$\mathrm{z}:=0$;

for $\mathrm{I}:=1$ to $\mathrm{zn}$ do

begin

if $\operatorname{pos}\left(\operatorname{copy}\left(\mathrm{s} 1, i^{*} \mathrm{sd}, \mathrm{sd}\right), \mathrm{s} 2\right)>0$ then inc $(\mathrm{z})$;

progressbar1.Position: $=\mathrm{ii}+\mathrm{i}$;

application.ProcessMessages;

end;

$\mathrm{x} 2:=\operatorname{int}((\mathrm{zn}-\mathrm{z}) / \mathrm{zn} * 10000) / 100$; 


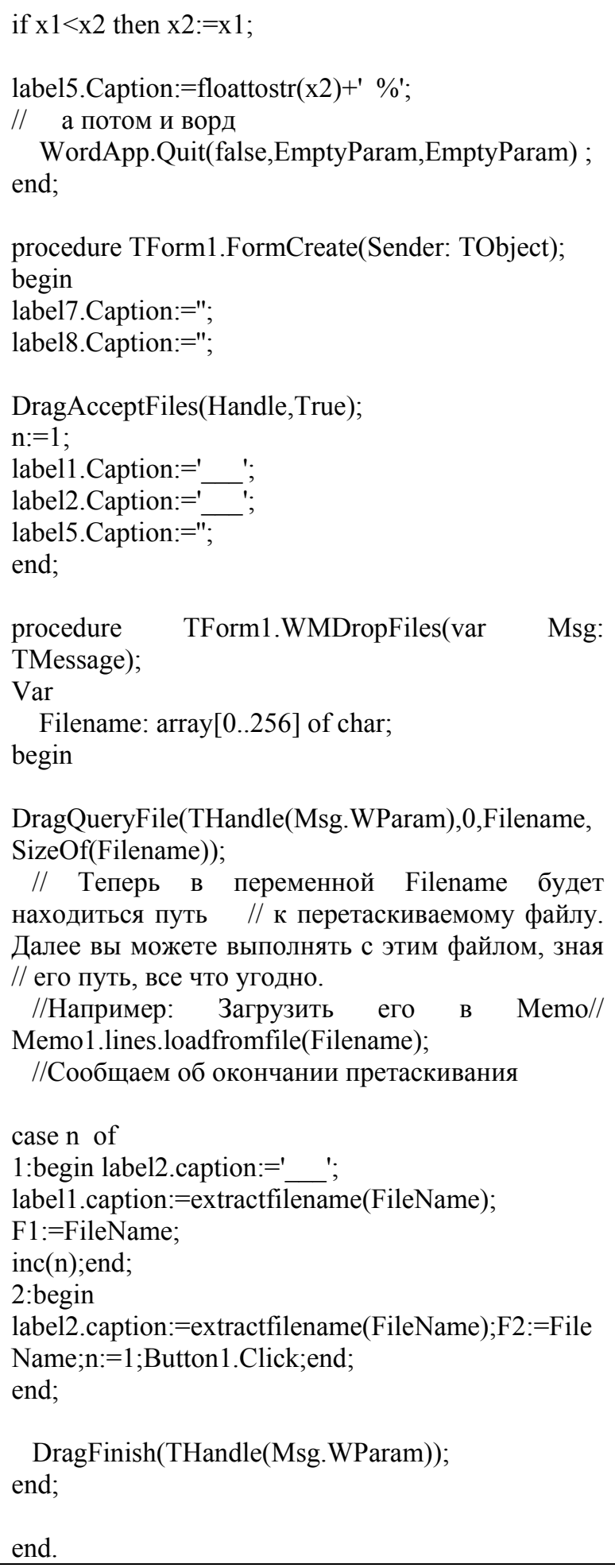

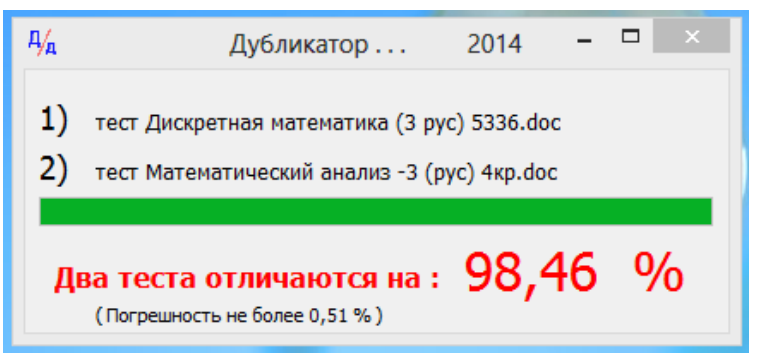

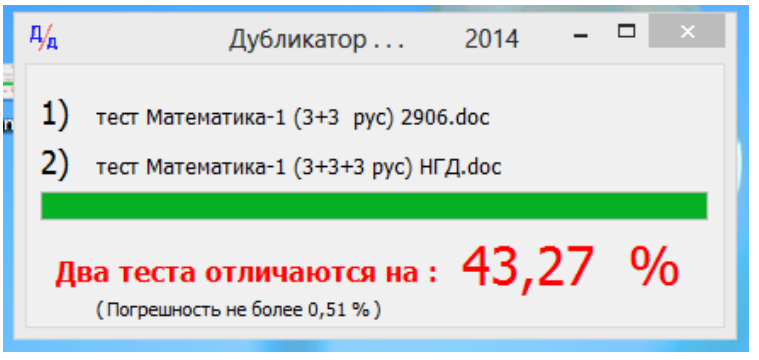

Рисунок 4 - Результаты анализа тестов.

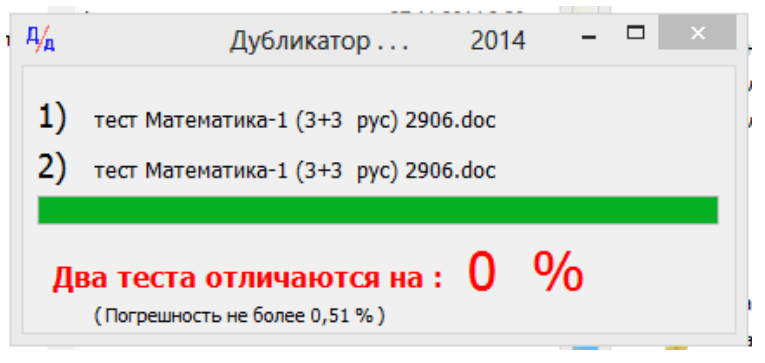

\section{Рисунок 5 - Сравнение одинаковых тестов.}

Разработанные алгоритмы и программа внедрена в производство с 2014 г. и прошла аппробацию в центре тестирования ТарГУ им.М.Х.Дулати.

\section{References:}

1. Shlahina S (2010) Tool for the rapid comparison of documents.. Available:
(Accessed:

ISPC European Technology in Science,

Malmö, Sweden 
2. (2014) Compare Suite Light - Freeware Compare Tool. Available: http://www.comparesuite.com/free-filecomparison.htm (Accessed: 27.02.2015).

3. (2015) Compare It! Available: http://www.grigsoft.com/ (Accessed: 27.02.2015).

4. (2015) Beyond Compare 3.1.9 Available: http://scootersoftware.com 27.02.2015).

5. (2015) WinMerge 2.12.4 Available: http://winmerge.org/ (Accessed: 27.02.2015).

6. (2015) Active File Compare 2.0 Available: http://www.formulasoft.ru/active-filecompare.html (Accessed: 27.02.2015).

7. (2015) Compare Spreadsheets for Excel 1.1.5 Available: https://www.mapilab.com/ru/excel/compare_spr eadsheets/ (Accessed: 27.02.2015).

8. (2015) Excel Compare 2.3 Available: http://www.formulasoft.com (Accessed: 27.02.2015).

9. (2015) xc Excel Compare Tools 1.0 Available: http://code.google.com/p/xcexcelcompare/down loads/detail?name $=$ xc.Excel.CompareTools.v1. 0.en.rar\&can $=2 \& q=$

10. (2015) Spreadsheet Compare 1.15 Available: http://www.lumadownload.com/ru/software/326 282/spreadsheet+compare +1.26 (Accessed: 27.02.2015). 